\title{
Author Correction: Selective inhibitors of mTORC1 activate 4EBP1 and suppress tumor growth
}

Bianca J. Lee, Jacob A. Boyer, G. Leslie Burnett (1D, Arun P. Thottumkara, Nidhi Tibrewal, Stacy L. Wilson, Tientien Hsieh, Abby Marquez, Edward G. Lorenzana, James W. Evans, Laura Hulea (1), Gert Kiss, Hui Liu, Dong Lee, Ola Larsson (1D, Shannon McLaughlan, Ivan Topisirovic, Zhengping Wang, Zhican Wang, Yongyuan Zhao, David Wildes, James B. Aggen, Mallika Singh, Adrian L. Gill, Jacqueline A. M. Smith (1) and Neal Rosen (D)

Correction to: Nature Chemical Biology https://doi.org/10.1038/s41589-021-00813-7, published online 24 June 2021.

In the version of this Article initially published, there were errors in Fig. 3b, Fig. 3c,d captions and in the main text.

Specifically, in Fig. 3b, the Time labels over lanes in the gels inadvertently duplicated labels from Fig. 3a. They have now been corrected to read, left-to-right, “48, 24, 16, 4, 4, 8, 16, 24, 48” (h).

The second sentence of Fig. 3c caption, originally reading "Data are the mean of technical duplicates," has been amended to read: "Data points are the mean of technical duplicates and representative of at least $n=2$ independent experiments." The caption for Fig. $3 \mathrm{~d}$, originally reading "d, Cell viability of MCF-7 rtTA3-4EBP1-4A cells treated with indicated concentrations of doxycycline for $3 \mathrm{~d}$, with error bars representing s.d. (representative of at least $n=2$ independent experiments). Each data point represents the mean cell viability of technical duplicates," has now been amended to read: "d, Cell viability of MCF-7 rtTA3-4EBP1-4A cells treated with indicated concentrations of doxycycline for $3 \mathrm{~d}$. Data points are the mean of technical duplicates and representative of at least $n=2$ independent experiments."

In the second paragraph of the Results section titled "Activation of 4EBP1 blocks cancer cell growth in vitro," seventh sentence, for the number of units reported in the sentence "sg4EBP1 cells were only three-fold less sensitive to RapaLink-1 compared to sgGFP cells, with RapaLink-1 suppressing proliferation at 0.3 and $0.1 \mathrm{nM}$ in sg4EBP1 and sgGFP cells, respectively," the numbers 0.3 and $0.1 \mathrm{nM}$ are now replaced with " 1 and 0.3 nM."

The changes have been made to the online version the article.

Published online: 6 October 2021

https://doi.org/10.1038/s41589-021-00905-4

() The Author(s), under exclusive licence to Springer Nature America, Inc. 2021 